\title{
A self-recalibration method based on scale-invariant registration for structured light measurement systems
}

\author{
Rui Chen ${ }^{\mathrm{a}, \mathrm{b}}$ Jing $\mathrm{Xu}^{\mathrm{a}}$ Song Zhang ${ }^{\mathrm{c}}$ Heping Chen ${ }^{\mathrm{d}}$ Yong Guan ${ }^{\mathrm{b}}$ Ken Chen ${ }^{\mathrm{a}}$ \\ ${ }^{a}$ State Key Laboratory of Tribology, and Department of Mechanical Engineering, Tsinghua University, Beijing, China, 100084. \\ ${ }^{\mathrm{b}}$ Beijing Advanced Innovation Center for Imaging Technology, Capital Normal University, Beijing, China, 100048. \\ ${ }^{\mathrm{c}}$ School of Mechanical Engineering, Purdue University, West Lafayette, Indiana,USA, 47907. \\ ${ }^{\mathrm{d}}$ Ingram School of Engineering, Texas State University, San Marcos, TX, USA, 78746.
}

\begin{abstract}
The measurement accuracy of structured light measurement depends on delicate offline calibration. However, in some practical applications, the system is supposed to be reconfigured so frequently to track the target that an online calibration is required. To this end, this paper proposes a rapid and autonomous self-recalibration method. For the proposed method, first, the rotation matrix and normalized translation vector are attained from the fundamental matrix; second, the scale factor is acquired based on scale-invariant registration such that the actual translation vector is obtained. Experiments have been conducted to verify the effectiveness of our proposed method, and the results indicate a high degree of accuracy.
\end{abstract}

Key words: Self-recalibration; 3D measurement; structured light

\section{Introduction}

The use of optical measurement techniques has increased in different areas in recent years. Compared with other methods, such as stereo vision[1], laser scanning[2], shape from motion[3], and the time of light[4] method, the structured light method has the advantages of low cost, small amounts of calculation, fast measurement speed, and high spatial resolution[5-8]. Thus, it is widely applied in many fields, such as workpiece inspection, motion tracking, and reverse engineering.

The structured light measurement system is composed of a projector and a camera. During the measurement, a set of encoded patterns is shot to the object by the projector, and the deformed patterns are captured and decoded by the camera. Finally, the 3D shape can be calculated using triangulation. The prerequisite of measurement is calibrating the intrinsic and extrinsic

\footnotetext{
* Corresponding author. E-mail address:jingxu@tsinghua.edu.cn (J.Xu)
}

Preprint submitted to optics and lasers in engineering parameters of the camera and projector. For most existing calibration methods for structured light measurement systems, an accurate calibration gauge or movement equipment is required; also, the relative position and orientation between the camera and the projector are supposed to be fixed[9,10].

However, in practical applications such as robot navigation, extrinsic parameters are desired to maximize the field of view (FOV), leading to continuous tracking of the measurement target. This action is similar to the phenomenon of two human eyes autonomously adjusting to view an object at different distances. Because the extrinsic parameters of the system change during the measurement procedure, the recalibration of extrinsic parameters needs to be performed online. Thus, the traditional offline calibration method based on a calibration gauge cannot be directly applied to this type of calibration.

Self-recalibration is a solution to the above problem$\mathrm{s}$ and has been widely studied recently. For the self- 
recalibration method, the intrinsic parameters of the system are often assumed to be known and constant, and only the extrinsic parameters are needed to be recalibrated. In Ref. [11], the extrinsic parameters are calculated using the corresponding point coordinates, and the solutions obtained by using different numbers of points are discussed. However, these corresponding points have to be non-planar, moreover, the scale factor is uncalibrated such that it cannot be used for accurate 3D reconstruction. In Ref. [12], a planar surface is used to obtain the relative position and orientation, where the plane constraint is used during the calculation. In the above methods, the translation vector between the projector and the camera is considered a normalized vector, and the scale factor is missing. Therefore, the size of the measurement target cannot be determined by the recalibration result. Moreover, a special calibration plane is also needed. In Ref. [13], the actual relative position and orientation can be attained, but the measurement system has only two degrees of freedom (DOFs); that is, the projector can move only along the $y$-axis and rotate around the z-axis. In Ref. [14], the extrinsic parameters are attained by measuring the object from two different positions and searching for point correspondence based on the texture of the object. This leads to large computational costs and ambiguities. In Ref.15, multiple cameras are used for a surplus measurement value, leading to increased installation complexity.

There is a strong demand for an online selfrecalibration method to autonomously and rapidly calibrate the extrinsic parameters without any extra calibration gauge, especially the translation vector. To this end, this paper proposes a self-recalibration method for the structured light measurement system based on scale-invariant registration. For the proposed method, first, the rotation matrix and the normalized translation vector are attained through the encoded pattern projection. Second, the scale factor is calibrated by registering the two point clouds of the same object with the scaleinvariant registration method. Finally, the actual size of the object can be determined by using the proposed calibration method. The rest of the paper is organized as follows: the mathematic model of the measurement system is described in Section 2. The calibration of the rotation matrix and normalized translation vector is described in Section 3. The scale factor acquisition is described in Section 4. The entire recalibration process, experiments, and results are shown in Section 5. In Section 6, our work is concluded.

\section{Mathematic models of camera and projector}

The structured light measurement system is composed of a projector and a camera. In this paper, a pinhole model is used for both the camera and the projector, where the projector is considered a pseudo-camera. The world coordinate system is coincident with the projector coordinate system, and the rotation matrix and translation vector from camera to projector are $R$ and $t$ as shown in Fig. 1.

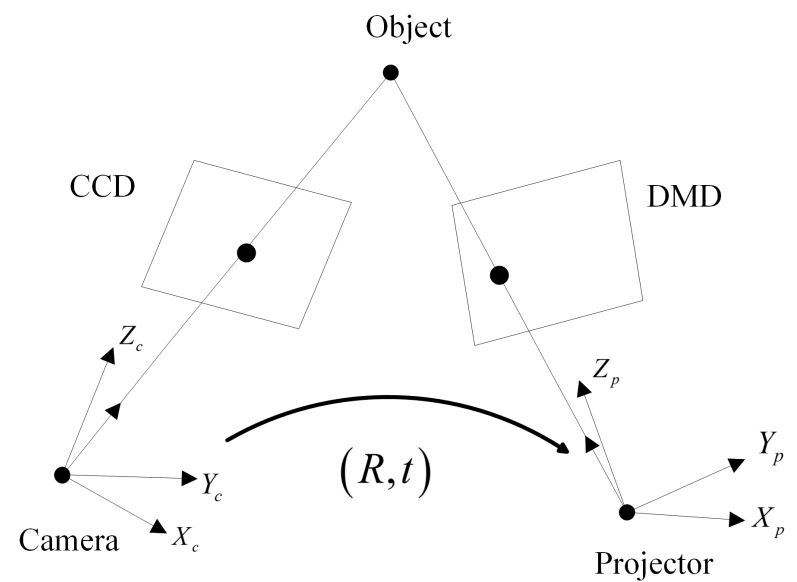

Fig. 1. Geometrical relations of the structured light measurement system.

For a 3D point $P$, its corresponding image pixel coordinates in the camera and the projector $m_{c}, m_{p}$ can be attained by:

$$
\begin{aligned}
& m_{c}=\alpha K_{c} P_{c} \\
& m_{p}=\beta K_{p} P_{p}
\end{aligned}
$$

where $P_{p}=P, P_{c}=R P_{p}+t, m_{c}=\left[u_{c}, v_{c}, 1\right]^{T}, m_{p}=$ $\left[u_{p}, v_{p}, 1\right]^{\mathrm{T}}, K_{c}=\left[\begin{array}{ccc}k_{x c} & 0 & o_{x c} \\ 0 & k_{y c} & o_{y c} \\ 0 & 0 & 1\end{array}\right], K_{p}=\left[\begin{array}{ccc}k_{x p} & 0 & o_{x p} \\ 0 & k_{y p} & o_{y p} \\ 0 & 0 & 1\end{array}\right]$ are the intrinsic parameter matrices of the camera and the projector, and $\alpha, \beta$ are non-zero scale coefficients.

Moreover, because of the lens distortion of the camera or the projector, the actual image pixel coordinates may derivate from the ideal ones, which can be expressed as[15]:

$$
\begin{aligned}
& u^{\prime}=\left(1+a_{0} r^{2}+a_{1} r^{4}+a_{2} r^{6}\right) u+s_{0} r^{2}+\left(p_{0}+p_{2} r^{2}\right)\left(r^{2}+2 u^{2}\right) \\
& v^{\prime}=\left(1+a_{0} r^{2}+a_{1} r^{4}+a_{2} r^{6}\right) v+s_{1} r^{2}+\left(p_{1}+p_{3} r^{2}\right)\left(r^{2}+2 v^{2}\right) \\
& r^{2}=u^{2}+v^{2}
\end{aligned}
$$


where $u, v$ are the ideal image pixel coordinates of the camera or the projector, $u^{\prime}, v^{\prime}$ are the actual image pixel coordinates, and $\left\{a_{i}\right\},\left\{s_{i}\right\},\left\{p_{i}\right\}$ are the lens distortion coefficients of the camera or the projector.

According to Eqs.(1) and (2), when $K_{c}, K_{p},\left\{a_{i}^{c}\right\}$, $\left\{s_{i}^{c}\right\},\left\{p_{i}^{c}\right\},\left\{a_{i}^{p}\right\},\left\{s_{i}^{p}\right\},\left\{p_{i}^{p}\right\}, R, t$ are known, the world $3 \mathrm{D}$ coordinate $P$ can be calculated from $m_{c}$ and $m_{p}$. Because intrinsic parameters $K_{c}, K_{p},\left\{a_{i}^{c}\right\},\left\{s_{i}^{c}\right\},\left\{p_{i}^{c}\right\}$, $\left\{a_{i}^{p}\right\},\left\{s_{i}^{p}\right\},\left\{p_{i}^{p}\right\}$ are determined by the lens and the C$\mathrm{CD}$ of the camera and the DMD of the projector, which are constant for one specific camera or projector, whereas extrinsic parameters $R, t$ are determined by the relative position and orientation between the camera and the projector, the calibration process of the structured light system is composed of two procedures:

The intrinsic parameters need to be calibrated only once for camera and projector. This is known as the static calibration procedure. The extrinsic parameters need to be recalibrated as many times as the position and orientation between the camera and the projector change. This is known as the dynamic recalibration procedure. The dynamic recalibration of extrinsic parameters is expected to be performed online rapidly and autonomously; that is, self-recalibration of extrinsic parameters is the important task of the structured light measurement system with varied extrinsic parameters. Thus, we focus on the extrinsic parameter self-recalibration in this paper.

\section{Rotation matrix and normalized translation vector calibration}

The position and orientation between the camera and the projector are contained in the fundamental matrix, which constrain the camera and projector pixel coordinates corresponding to a common physical $3 \mathrm{D}$ point. To recalibrate the extrinsic parameters, we first obtain the fundamental matrix through encoded pattern projection. Second, the rotation matrix and normalized translation vector between the camera and the projector are further decomposed from the fundamental matrix.

\subsection{Fundamental matrix calculation}

The relation between the object point coordinate $P_{c}$ in camera coordinate frame and $P_{p}$ in projector coordinate frame can be expressed as:

$$
P_{c}=R P_{p}+t
$$

yielding to

$$
P_{c}-t=R P_{p}
$$

And because the cross production of two vectors is perpendicular to both vectors, the following equation could be obtained:

$$
\left(P_{c}-t\right)^{T}\left(t \times P_{c}\right)=0
$$

Combining Eqs.(3) and (4), the constraint between $P_{c}$ and $P_{p}$ can be obtained as:

$$
P_{p}^{T} R^{T} S_{t} P_{c}=0
$$

where $S_{t}$ is the skew symmetric matrix of translation vector $t: S_{t}=\left[\begin{array}{ccc}0 & -t_{3} & t_{2} \\ t_{3} & 0 & -t_{1} \\ -t_{2} & t_{1} & 0\end{array}\right]$

According to Eq(1), the unknown actual physic 3D coordinates $P_{c}, P_{p}$ in (6) can be substituted by the corresponding image pixel coordinates $m_{c}, m_{p}$.

For the structured light measurement system, the encoded vertical and horizontal fringe patterns are successively shot to the measured object. Then, for every camera pixel coordinate $m_{c}$, the corresponding projector pixel coordinate $m_{p}$ is obtained by decoding the captured patterns. Therefore, the correspondence $m_{c}, m_{p}$ for a physical 3D point $P$ can be found.

Thus, Eq.(6) becomes:

$$
m_{p}\left(K_{p}^{-1}\right)^{T} R^{T} S_{t} K_{c}^{-1} m_{c}=0
$$

Fundamental matrix $F$ is defined as:

$$
F=\left(K_{p}^{-1}\right)^{T} R^{T} S_{t} K_{c}^{-1}
$$

Eq.(7) becomes:

$$
m_{p} F m_{c}=0
$$

The fundamental matrix $F$ is of rank 2 , and it has 8 parameters. It is noted that we can obtain only a normalized fundamental matrix $F$ from Eq.(8); that is, the actual fundamental matrix is perhaps a scale factor by the solved normalized fundamental matrix.

To enhance the robustness of the algorithm, a large number of corresponding point pairs are taken into account, and the random sample consensus algorithm (RANSAC))[16] is used to optimize the calculation result. For this method, a random subset of 8 point pairs is extracted from all of the points iteratively to calculate the fundamental matrix $F$. The particular solution closest to the average of all of the solutions is taken as the final solution.

Once the normalized fundamental matrix is attained, rotation matrix $R$ and normalized translation vector $t$ between the camera and the projector can be further recovered from $F$, as described in Section 3.2. 


\subsection{Recovery of rotation matrix and normalized translation vector}

Define essential matrix $E=R^{T} S_{t}$. Because normalized fundamental matrix $F$ is attained as described above, $K_{c}$ and $K_{p}$ are attained from the static calibration, essential matrix $E$ can be calculated by:

$$
E=K_{p}^{T} F K_{c}
$$

Then, $R$ and $t$ can be recovered from $E$ by the following processes:

The SVD (singular value decomposition) of $E$ is:

$$
E=U \sum V^{T}
$$

where $\Sigma=\left[\begin{array}{ccc}\varepsilon_{1} & 0 & 0 \\ 0 & \varepsilon_{2} & 0 \\ 0 & 0 & 0\end{array}\right]$, and $\operatorname{det}(U)>0, \operatorname{det}(V)>0$.

Then, $t=\eta\left[u_{13}, u_{23}, u_{33}\right]^{T}$, and $R=U D V^{T}$ or $R^{\prime}=$ $U D^{T} V^{T}$, where $\eta$ is the unknown scale factor, and $D=\left[\begin{array}{ccc}0 & 1 & 0 \\ -1 & 0 & 0 \\ 0 & 0 & 1\end{array}\right]$

Of note, the scale factor $\eta$ is impossible to determine using this method.

Because of the inherent ambiguities, there are four possible solutions of the combination of $R$ and $t$ : $[R, t],[R,-t],\left[R^{\prime}, t\right],\left[R^{\prime},-t\right]$. The chirality constraint is imposed to eliminate the invalid solutions and find the true solution.

For one arbitrary pair of corresponding image pixel points $m_{c}$ and $m_{p}$, the $3 \mathrm{D}$ coordinates in the camera coordinate system and the projector system $P_{c}{ }^{\prime}$ and $P_{p}{ }^{\prime}$ are calculated by using $R$ and $t . P_{c}^{\prime}$ and $P_{p}{ }^{\prime}$ have to be in the common FOV of the camera and the projector.

However, the scale factor $\eta$ which is necessary for the reconstruction of the actual size of the object, as shown in Fig. 2, is still unknown. Note that the small object is the reconstruction result with the normalized translation vector, whereas the large object is the result with the scale factor $\eta$. Different scale factors between the projector and the camera would lead to different translation vectors, so determining the scale factor is necessary for determining the size of the object. To this end, a scale-invariant $3 \mathrm{D}$ point cloud registration is applied to attain $\eta$ in this paper.

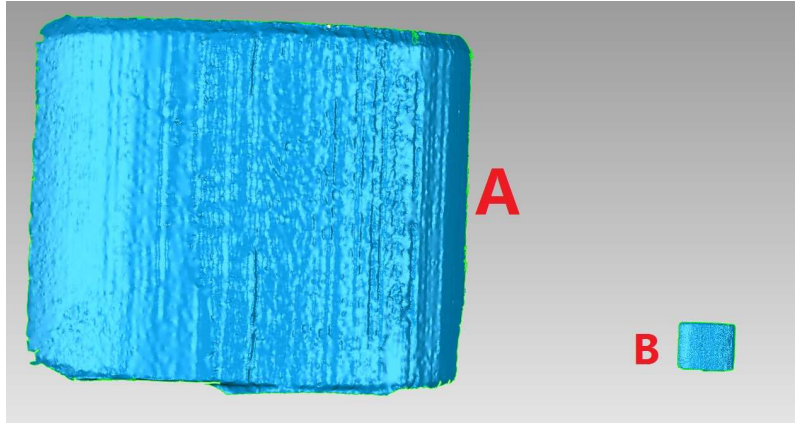

Fig. 2. Reconstruction result comparison:A-reconstruction result with the actual translation vector; B-reconstruction result with the normalized translation vector.

\section{Scale factor acquisition}

In this section, the scale factor acquisition by using a scale-invariant point cloud registration method is proposed to determine the actual translation vector. The basic concept is that some common features of the measured object are extracted to establish the connection between the origin system and the successive reconfigured system so that the scale factor for every reconfigured system can be attained.

The continuous measured target object is monitored by the original system and the reconfigured system. The two measured point clouds are registered, and the scale factor between them is attained. Moreover, because the exact extrinsic parameters of the original system are calibrated from static calibration procedure, the scale factor of the reconfigured system can be acquired. However, because the scale factor of the reconfigured system is unknown and the normalized translation vector is used for the reconfigured system, the two measured point clouds are in different scales. Thus, the conventional registration methods, such as FPFH[17], VFH[18], NARF[19], and 4-PCS[20], may not work in this situation. To this end, some features invariant to scale are extracted from the point cloud for registration and further scale factor acquisition.

\subsection{SIFT feature detection}

The Scale Invariant Feature Transform (SIFT) feature detector has been proposed as a robust 2D image feature detection algorithm[21]. The SIFT algorithm is described as follows:

First, the image is convolved with a series of Gaussian filters of different sizes, and then the Difference of Gaussian (DoG) function is performed. The points with local maximum or minimum results are considered can- 
didates for key points. Second, points within low contrast neighborhoods and points at edges are excluded as candidates. Finally, the histogram calculated from the gradient magnitudes in all directions is generated as the feature descriptor.

The features extracted from the SIFT detector are invariant to scale and rotation, thus making them compatible for application in a correspondence search for the $3 \mathrm{D}$ point cloud registration and further scale factor acquisition.

\subsection{D point cloud preprocess}

The SIFT algorithm works only with 2D images; thus, some point cloud preprocess need to be performed before using the SIFT algorithm for 3D feature correspondence searching:

First, for the structured light measurement system, the $3 \mathrm{D}$ coordinates $\{P\}$ are calculated from the camera image pixel coordinates $\left\{m_{c}\right\}$ and their corresponding projector image pixel coordinates $\left\{m_{p}\right\}$. A 2D matrix $M_{D}$ of the same size as the camera resolution is generated by setting the value at each point as the distance between $P$ and the origin:

$$
M_{D}\left(m_{c}\right)=\sqrt{x_{P}^{2}+y_{P}^{2}+z_{p}^{2}}
$$

Second, $M_{D}$ is scaled to $0-255$, with the maximum distance scaled to 255 , and the others scaled the same. After the preprocess, $M_{D}$ is attained and ready for the SIFT feature detection.

\subsection{Scale factor acquirement}

The same target object is first measured by the structured light system which has been calibrated statically, and the 2D matrix $M_{D}^{(0)}$ is generated. Then, the measurement system is reconfigured and recalibrated. The target is measured by the system again, and $M_{D}^{(1)}$ is generated.

The SIFT feature descriptors are built for $M_{D}^{(0)}$ and $M_{D}^{(1)}$. Then, to attain the scale factor between the two point clouds, the registration between them is performed by searching for corresponding key points in $M_{D}^{(0)}$ and $M_{D}^{(1)}$.

For two point clouds in different scales $\{Q\}$ and $\left\{Q^{\prime}\right\}$, the transformation between them is described as follows:

$$
Q^{\prime}=\varepsilon R_{Q} Q+t_{Q}
$$

where $R_{Q}, t_{Q}$ are the rotation matrix and translation vector, respectively, and $\varepsilon$ is the scale factor.
The registration process is as follows:

For a set of correspondences $\{q\}$ and $\left\{q^{\prime}\right\}$, first, the covariance matrices are built:

$$
\begin{aligned}
& \mathrm{E}=\operatorname{cov}(q) \\
& \mathrm{E}^{\prime}=\operatorname{cov}\left(q^{\prime}\right)
\end{aligned}
$$

Second, the principal components analysis (PCA) for $\mathrm{E}$ and $\mathrm{E}^{\prime}$ is performed, and $\left\{\lambda_{i}\right\},\left\{\lambda_{i}^{\prime}\right\}(i=1,2,3)$ are the eigenvalues for $\mathrm{E}$ and $\mathrm{E}^{\prime}$, respectively.

Finally, the scale factor $\varepsilon$ can be attained by:

$$
\varepsilon=\sqrt{\lambda_{1}^{\prime} / \lambda_{1}}
$$

where $\lambda_{1}, \lambda_{1}{ }^{\prime}$ are the maximum eigenvalues.

After $\varepsilon$ is attained, $R_{Q}, t_{Q}$ can be calculated by using the conventional rigid transformation estimation.

However, because there are many inaccurate matches, which would lead to incorrect scale factor results, the RANSAC algorithm is applied to enhance the robustness of the registration.

For all of the corresponding key points, a set of point pairs are selected randomly, and the transformation matrix and scale factor between two point clouds are attained. Then, the registration error is calculated by computing the point distance between the two point clouds. The processes are repeated a number of times, and the registration with the smallest error is chosen as the final registration, whose scale factor is taken as the final scale factor.

After the point cloud registration, the scale factor between the two point clouds $\varepsilon$ is attained. Furthermore, the scale factor $\eta$ of the recalibrated translation vector is attained, which means that the physical relative position and orientation of the camera and the projector have been acquired, thus accomplishing the entire self-recalibration. The result would be applied for the reconfigured measurement system.

\section{Experiments and results}

To verify the feasibility and accuracy of the proposed method, a structured light measurement system is constructed. The system is composed of a digital projector and a camera. The projector is a DLP Pro 4500 produced by Texas Instruments (TI,Dallas Metroplex, Texas, USA) with a resolution of $912 \times 1140$. The camera is a JAI GO-5000M-USB(JAI, Sakae-chou Kanagawa-ku, Yokohama,Japan), with a resolution of $2560 \times 2048$.

First, the measurement system is calibrated statically with a calibration board, as shown in Fig. 3. The board 
is composed of $9 \times 11$ circular dots, 5 of which are large dots with a diameter of $15 \mathrm{~mm}$ used for world coordinate system definition; the remaining dots are small dots with a diameter of $7.5 \mathrm{~mm}$ as the calibration features.

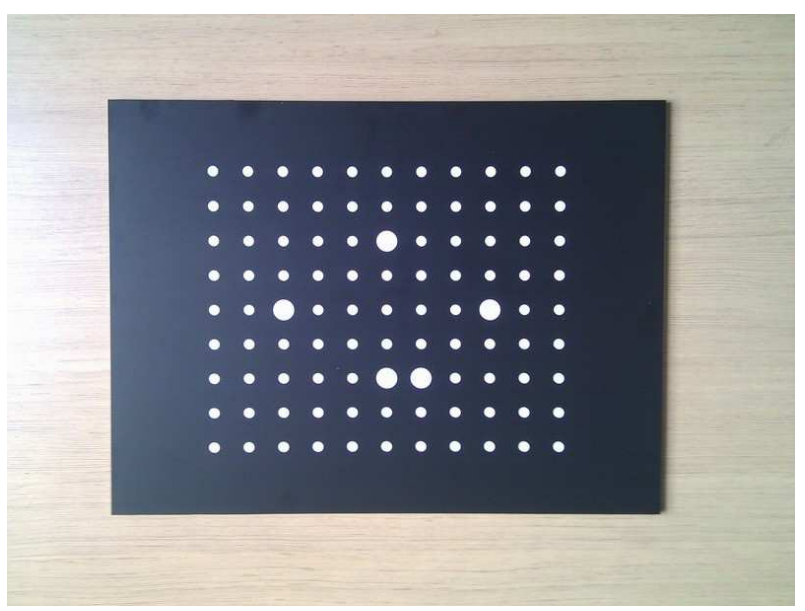

Fig. 3. Calibration board for static calibration

\subsection{Static calibration procedure}

The static calibration procedure is as follows:

(1) The calibration board is placed at different positions and orientations, which are captured by the camera. The camera image pixel coordinates of the reference dots are attained using sub-pixel edge detection and ellipse fitting.

(2) The horizontal and vertical fringe patterns are successively shot to the calibration board, and the projector image pixel coordinates of the reference dots are attained by using dual-frequency phase shifting decoding.

(3) The intrinsic parameters of the camera and the projector are calibrated using Zhangs method[22].

(4) The rotation matrix $R_{0}$ and the translation vector $t_{0}$ between the projector and the camera are attained using RANSAC optimization.

After the static calibration, the target object, as shown in Fig. 4, is measured. Then, the measurement system is reconfigured where the position and orientation of the camera are changed to keep the target object in the FOV of the camera.

\subsection{Self-recalibration procedure}

The dynamic self-recalibration procedure is performed as follows:

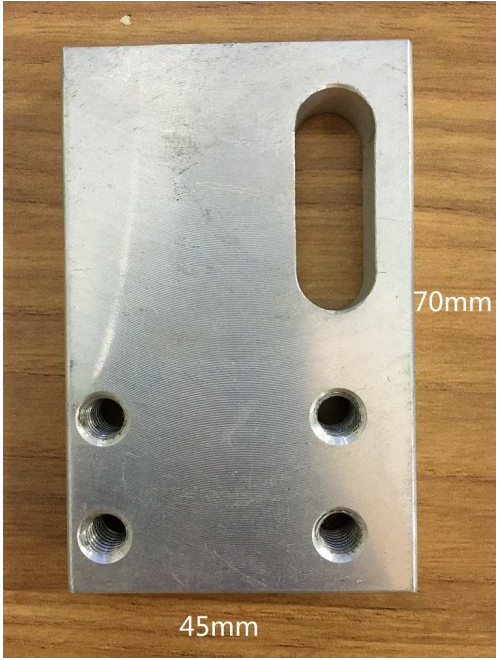

Fig. 4. Measured target object.

(1) The horizontal and vertical fringe patterns are successively shot to the target object, and the corresponding camera and projector image pixel coordinates $\left\{m_{c}\right\},\left\{m_{p}\right\}$ are attained.

(2) The rotation matrix $R_{1}$ and the normalized translation vector $t_{1}$ are attained, using the method described in Section 3.

(3) The 3D point cloud $\left\{P_{1}\right\}$ of the displaced target object is calculated using $R_{1}$ and $t_{1}$.

(4) The 2D distance matrices $M_{D}^{(0)}$ and $M_{D}^{(1)}$ are generated.

(5) The scale invariant registration method based on SIFT is performed. The correspondence search result is shown in Fig. 5, and the scale factor $\eta$ is acquired. The entire self-recalibration process is shown in Fig. 6. Despite there being many inaccuracies, the correct scale factor $\eta$ is attained using the RANSAC algorithm, where the correct correspondences are found and used for calculation.

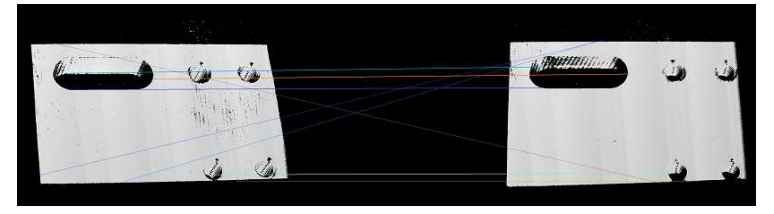

Fig. 5. Correspondence search result.

After the self-recalibration, the new transformation matrix $M_{1}$ between the camera and the projector is generated:

$$
M_{1}=\left[\begin{array}{cc}
R_{1} & \eta t_{1} \\
0 & 1
\end{array}\right]
$$




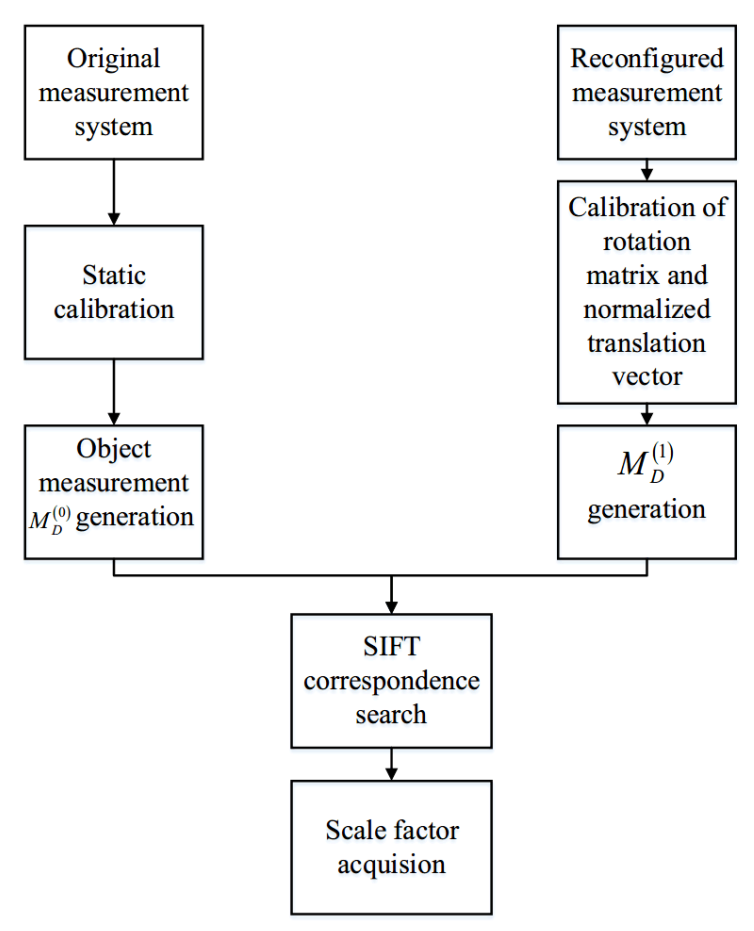

Fig. 6. Self-recalibration process.

which can be used for the reconfigured measurement system.

\subsection{Experimental results}

In order to demonstrate the robustness and accuracy of the proposed method, the system has been reconfigured 30 times by using different target objects, and for each configuration it is dynamically self-recalibrated without any calibration gauge and statically calibrated with the calibration gauge.

First, the direction deviations of translation matrix and rotation vector between static calibration and proposed self-recalibration are measured in radians. The deviation angle for the rotation matrix is defined as[23]:

$$
\alpha_{\text {err }}=\frac{1}{3} \sum_{i=1,2,3} \operatorname{acos}\left(\left(R_{1} e_{i}\right)^{T} R_{2} e_{i}\right)
$$

where $e_{i}(i=1,2,3)$ represents the unit vector for three axis. And the statics are shown in Table.1.

Table 1

Direction deviation of translation and rotation

\begin{tabular}{ccc} 
& mean & std.var \\
\hline $\mathrm{R}(\mathrm{rad})$ & 0.0166 & $1.76 \mathrm{e}-4$ \\
\hline $\mathrm{T}(\mathrm{rad})$ & 0.1370 & 0.0030 \\
\hline
\end{tabular}

Table 2

The measurement distance between planes.

\begin{tabular}{ccccc}
\hline \multirow{2}{*}{$\begin{array}{c}\text { Distance } \\
\text { value(mm) }\end{array}$} & $\begin{array}{c}\text { Static } \\
\text { valibration(mm) }\end{array}$ & $\begin{array}{c}\text { Self-recalibration Self-recalibration } \\
(\mathrm{mm})\end{array}$ & Error \\
\hline $\mathrm{d}_{12}$ & 15.00 & 15.38 & 15.51 & $3.4 \%$ \\
$\mathrm{~d}_{23}$ & 19.88 & 20.40 & 20.31 & $2.67 \%$ \\
$\mathrm{~d}_{34}$ & 25.16 & 25.42 & 25.76 & $2.38 \%$ \\
$\mathrm{~d}_{45}$ & 30.17 & 30.51 & 30.37 & $0.66 \%$ \\
\hline
\end{tabular}

Second, the scale factors are compared. The mean error is $1.605 \%$, with a standard deviation of $0.74 \%$.

A brick with 5 stairs as shown in Fig. 7 is measured by the reconfigured system, and the reconstruction results are shown in Fig. 8. The measurement results are given in Table. 2, where $d_{i j}$ represents the distance between plane $i$ and plane $j$. The mean error is $2.34 \%$, which is smaller than $3.31 \%$ in [24]. Then a statue is measured and the reconstruction results obtained by proposed method and static calibration method are shown in Fig.9.

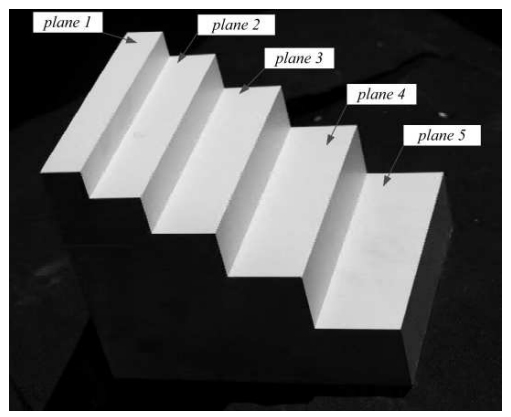

Fig. 7. Test brick

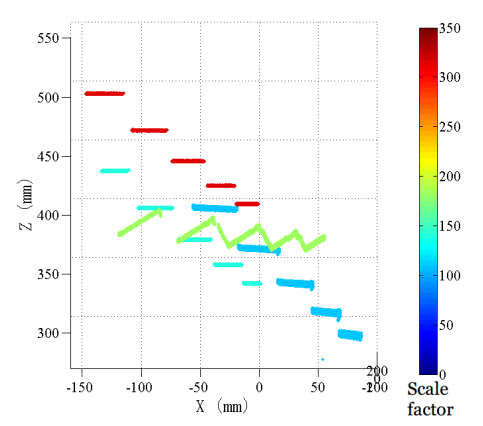

Fig. 8. Reconstruction results of the brick

The comparison and measurement results demonstrate that the proposed self-recalibration method can lead to accurate results. 


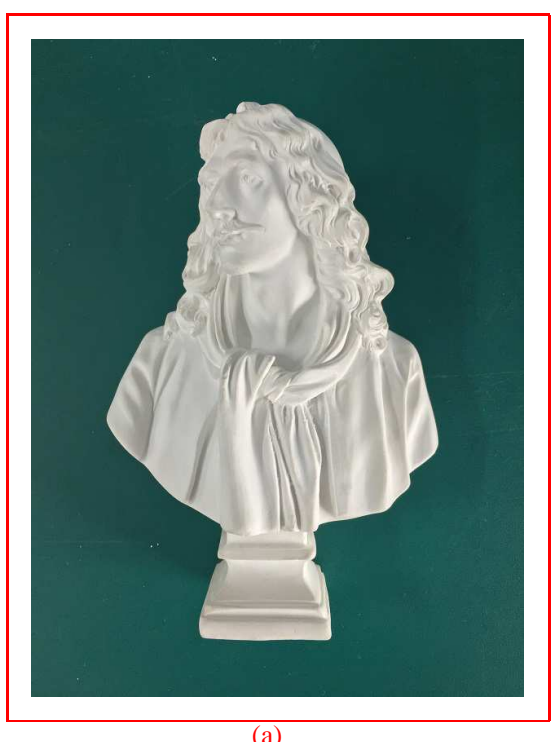

(a)

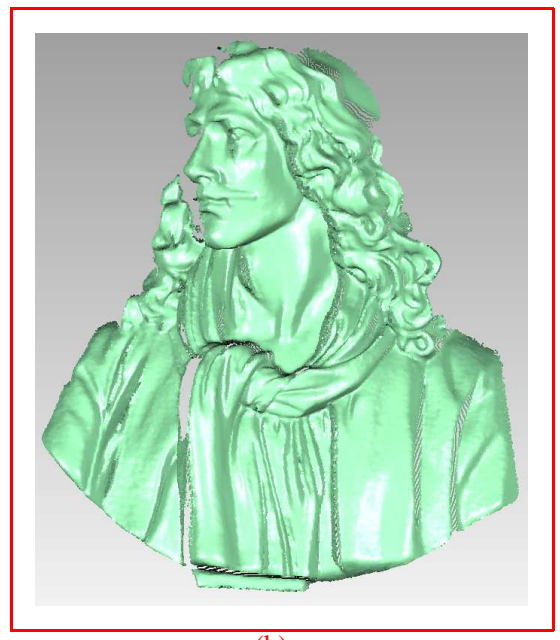

(b)

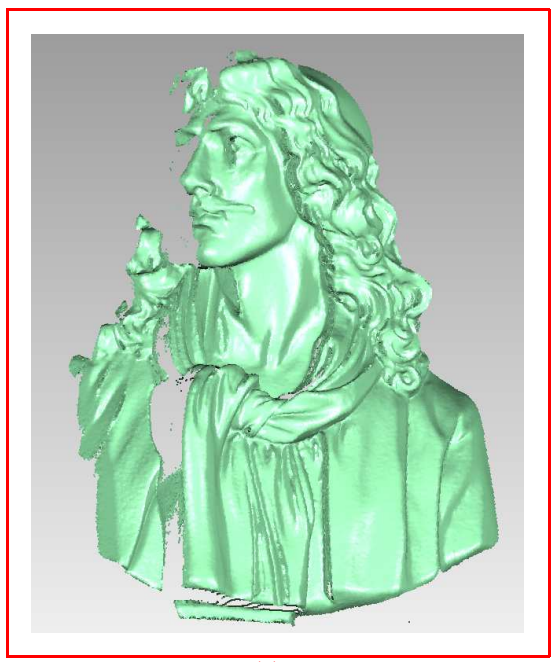

(c)

Fig. 9. Comparison of reconstruction results: (a) measurement object; (b) proposed method; (c) static calibration method.

\subsection{Discussion}

The recalibration accuracy is composed of two part$\mathrm{s}$ : the accuracy of the rotation matrix and translation vector, and the accuracy of scale factor. First, the accuracy of the rotation matrix and translation vector depends on the fundamental matrix calculation, which is determined by the decoding accuracy of fringe patterns. Thus, a dual-frequency phase shifting method with a large phase shift number like 19 is applied to eliminate the influence of ambient light and improve the decoding accuracy. Furthermore, the RANSAC strategy is used to exclude the outliner points.

Second, for the scale factor acquisition, because the final result is chosen by comparing the registration error, when the calculated scale factors are close to the real scale factor, the registration errors are close and the scale factor closest to the real one may not be chosen. According to experimental results, the distance between camera and projector would not influence the recalibration accuracy. For the orientation of the target object, the accuracy is not influenced when there are enough correct corresponding keypoints for registration.

\section{Conclusions}

Accounting for fast speed, low cost, and high accuracy, the structured light measurement has been widely studied and applied in industrial manufacturing. However, in some practical applications, the measurement system has to be reconfigured frequently to track the target object, and the recalibration has to be performed online. The conventional calibration method is timeconsuming, and an accurate equipment is needed. In this paper, a rapid and autonomous self-recalibration method is proposed to overcome this problem. In the proposed method, first, the rotation matrix and the normalized translation vector are attained through the encoded pattern projection; second, the scale factor is acquired by using a scale-invariant point cloud registration method. Experimental results demonstrate the effectiveness and accuracy of our proposed method. The proposed method can be applied in the structured light measurement with varied extrinsic parameters.

\section{Acknowledgement}

The work is partially supported by National Science Foundation of China (No. 51105218); and the Fund of State Key Laboratory of Tribology of China( No.SKLT2015B10). 


\section{References}

[1] Qi, L., et al., Quality inspection guided laser processing of irregular shape objects by stereo vision measurement: application in badminton shuttle manufacturing. Optical Engineering, 2015. 54(11): p. 113101-113101.

[2] Buckner, B.D., et al., Laser-scanning structural health monitoring with wireless sensor motes. Optical Engineering, 2008. 47(5): p. 054402-054402-9.

[3] Shi, J., et al., Reconstruction of dense three-dimensional shapes for outdoor scenes from an image sequence. Optical Engineering, 2013. 52(12): p. 123104-123104.

[4] Cui, Y., et al. 3D shape scanning with a time-of-flight camera. in Computer Vision and Pattern Recognition (CVPR), 2010 IEEE Conference on. 2010: IEEE.

[5] Forster, F. A high-resolution and high accuracy realtime $3 \mathrm{~d}$ sensor based on structured light. in 3D Data Processing, Visualization, and Transmission, Third International Symposium on. 2006: IEEE.

[6] Ishii, I., K. Yamamoto and T. Tsuji. High-speed 3D image acquisition using coded structured light projection. in Intelligent Robots and Systems, 2007. IROS 2007. IEEE/RSJ International Conference on. 2007: IEEE.

[7] Zhang, S. and S. Yau, Three-dimensional shape measurement using a structured light system with dual cameras. Optical Engineering, 2008. 47(1): p. 013604-013604-12.

[8] Salvi, J., et al., A state of the art in structured light patterns for surface profilometry. Pattern recognition, 2010. 43(8): p. 2666-2680.

[9] Li, Z., et al., Accurate calibration method for a structured light system. Optical Engineering, 2008. 47(5): p. 053604-053604-9.

[10] Chen, X., et al., Accurate calibration for a cameraCprojector measurement system based on structured light projection. Optics and Lasers in Engineering, 2009. 47(3): p. 310-319.

[11] Philip, J., Critical point configurations of the 5-, 6-, 7-, and 8-point algorithms for relative orientation. 1998: Department of Mathematics, Royal Institute of Technology Stockholm, Sweden.

[12] Zhang, B., Y.F. Li and Y.H. Wu, Self-recalibration of a structured light system via plane-based homography. Pattern Recognition, 2007. 40(4): p. 1368-1377.

[13] Li, Y.F. and S.Y. Chen, Automatic recalibration of an active structured light vision system. Robotics and Automation, IEEE Transactions on, 2003. 19(2): p. 259-268.

[14] Tian, J., Y. Ding and X. Peng, Self-calibration of a fringe projection system using epipolar constraint. Optics \& Laser Technology, 2008. 40(3): p. 538-544.

[15] Vo, M., et al., Advanced geometric camera calibration for machine vision. Optical Engineering, 2011. 50(11): p. 110503110503-3.

[16] Fischler, M.A. and R.C. Bolles, Random sample consensus: a paradigm for model fitting with applications to image analysis and automated cartography. Communications of the ACM, 1981. 24(6): p. 381-395.

[17] Rusu, R.B., N. Blodow and M. Beetz. Fast point feature histograms (FPFH) for 3D registration. in Robotics and Automation, 2009. ICRA'09. IEEE International Conference on. 2009: IEEE.

[18] Rusu, R.B., et al. Fast 3d recognition and pose using the viewpoint feature histogram. in Intelligent Robots and Systems (IROS), 2010 IEEE/RSJ International Conference on. 2010: IEEE.
[19] Steder, B., et al. NARF: 3D range image features for object recognition. in Workshop on Defining and Solving Realistic Perception Problems in Personal Robotics at the IEEE/RSJ Int. Conf. on Intelligent Robots and Systems (IROS). 2010.

[20] Silva Jr, J.P.D., D.L. Borges and F. De Barros Vidal. A dynamic approach for approximate pairwise alignment based on 4-points congruence sets of 3D points. in Image Processing (ICIP), 2011 18th IEEE International Conference on. 2011: IEEE.

[21] Lowe, D.G., Distinctive image features from scale-invariant keypoints. International journal of computer vision, 2004. 60(2): p. 91-110.

[22] Zhang, Z., A flexible new technique for camera calibration. Pattern Analysis and Machine Intelligence, IEEE Transactions on, 2000. 22(11): p. 1330-1334.

[23] Rodehorst, V., Heinrichs, M., \& Hellwich, O. (2008). Evaluation of relative pose estimation methods for multi-camera setups. International Archives of Photogrammetry and Remote Sensing (ISPRS08), 135-140.

[24] Li, Y.F. and B. Zhang, Toward dynamic recalibration and threedimensional reconstruction in a structured light system. Journal of the Optical Society of America A, 2007. 24(3): p. 785-793. 